\title{
Finite element numerical investigation into unsteady MHD radiating and reacting mixed convection past an impulsively started oscillating plate
}

\author{
P. M. Matao ${ }^{1}$, B. Prabhakar Reddy ${ }^{1}$, J. M. Sunzu ${ }^{1}$ and O. D. Makinde ${ }^{2}$ \\ ${ }^{1}$ Department of Mathematics and Statistics, CNMS, The University of Dodoma, P.O. Box No. 338, Dodoma, TANZANIA \\ ${ }^{2}$ Faculty of Military Science, Stellenbosch University, Private Bag X2, Saldanha 7395, SOUTH AFRICA \\ *Corresponding Author's e-mail: prabhakar.bijjula@gmail.com, Tel +91-9885544480 \\ ORCID iD: http://orcid.org/ 0000-0003-0217-5923
}

\begin{abstract}
In this article, numerical investigation is carried out for the unsteady MHD mixed convection flow of radiating and chemically reacting fluid past an impulsively started oscillating vertical plate with variable temperature and constant mass diffusion. The transport model employed includes the Hall current. A uniform magnetic field is applied transversely to the direction of the fluid flow. The flow consideration is subject to small magnetic Reynolds number. The Rosseland approximation is used to describe the radiation heat flux in the energy equation. The dimensionless governing system of partial differential equations of the flow has been solved numerically by employing the finite element method. The influence of pertinent parameters on primary velocity, secondary velocity, temperature and concentration are presented graphically whereas primary skin friction, secondary skin friction, Nusselt number and Sherwood number are presented in tabular form. A comparison of the present method was made with the exact solution obtained by Rajput and Kanaujia (2016) by considering primary and secondary skin frictions, it was noticed that a very good agreement.
\end{abstract}

Keywords: MHD, radiation parameter, chemical reaction parameter, magnetic parameter, Hall current.

DOI: http://dx.doi.org/10.4314/ijest.v12i1.4

\section{Introduction}

The study of MHD with heat and mass diffusion in the presence of radiation has attracted the attention of a large number of researchers due to its diverse applications. Many processes in new engineering occur at high temperature and knowledge of radiation heat transfer becomes necessary for the design of the pertinent equipment. Nuclear power plants, gas turbines and the various propulsion devices for aircraft, missiles, satellites, and space vehicles, etc are examples of such engineering areas. Muthucumaraswamy and Janakiraman (2006) studied MHD and radiation effects on moving isothermal vertical plate with variable mass diffusion. Rajesh and Varma (2010) presented radiation effects on MHD flow through a porous medium with variable temperature and mass diffusion. Mebine and Adigio (2011) analyzed thermal radiation effects on transient MHD free convection flow over a vertical surface embedded in a porous medium with periodic boundary conditions. Rajput and Kumar (2017) investigated radiation effect on MHD flow past an inclined plate with variable temperature and mass diffusion. Nandkeolyar et. al (2013) studied unsteady hydro-magnetic natural convection flow of a dusty fluid past an impulsively moving vertical plate with ramped temperature in the presence of thermal radiation. Thermo-dynamic analysis of unsteady MHD mixed convection with slip and thermal radiation over a permeable surface were reported by Muhammad and Makinde (2017).

In all the above cited studies, the effects of chemical reaction on the flow are not taken into account. Investigation of MHD flow with chemical reaction is of much significance due to its wide applications in many branches of science and engineering. In many chemical engineering processes, chemical reaction takes place between a foreign mass and the working fluid in which the plate is moving. These processes take place in numerous industrial applications such as manufacturing of ceramics or glassware, polymer production and food processing. In nature, the presence of pure air or water is not possible. Some foreign mass may be present 
either naturally or mixed with the air or water. The presence of a foreign mass in air or water causes some kind of chemical reaction. Theoretical study of chemical reaction effects on vertical oscillating plate with variable temperature were presented by Muthucumaraswamy and Meenakshisundaram (2006). Muthucumaraswamy and Janakiraman (2008) examined the mass transfer effects on isothermal vertical oscillating plate in the presence of chemical reaction. Mahapatra et. al (2010) analyzed the effects of chemical reaction on free convection flow through a porous medium bounded by a vertical surface. Rajesh and Varma (2010) investigated the chemical reaction effects on free convection flow past an exponentially accelerated vertical plate. Effects of chemical reaction on the unsteady free convection flow past an infinite vertical permeable moving plate with variable temperature was analyzed by El-Fayez (2012). Sehkar and Reddy (2012) presented the effects of chemical reaction on MHD free convective oscillatory flow past a porous plate with viscous dissipation and heat sink. Makinde (2012) studied chemically reacting hydromagnetic unsteady flow of radiating fluid past a vertical plate with constant heat flux. Jayakar et. al (2018) investigated thermodiffusion effects on MHD chemically reacting fluid flow past an inclined porous plate in a slip flow regime.

However, in all these investigations, the effects of Hall current are not taken into account. The effect of Hall current cannot be completely ignored if the strength of the magnetic field is strong and number of density electrons is small as it is responsible for the charge of the flow pattern of an ionized gas. Hall current results in a development of an additional potential difference between opposite surfaces of a conductor for which a current is induced perpendicular to both the electric and magnetic field. This current is known as Hall current. It plays an important role in determining features of the fluid flow problems because it induces secondary flow in the flow field. Sharma et. al (2007) analyzed the effects of Hall current on MHD mixed convection flow of a viscous incompressible fluid past a vertical porous plate immersed in a porous medium with heat source/sink. Maguna and Mutua (2013) presented the Hall current effects on free convection flow and mass transfer past a semi infinite vertical flat plate. Hall and rotation effects on MHD flow past an exponentially accelerated vertical plate with combined heat and mass transfer effects were analyzed by Thamizhsudar et. al (2015). MHD flow past a vertical plate with variable temperature and mass diffusion in the presence of Hall current was studied by Rajput and Kanaujia (2016). Recently, Reddy (2018) presented Hall effect on MHD transient flow past an impulsively started infinite horizontal porous plate in a rotating system.

The objective of the present work is to study the unsteady MHD mixed convection flow past an impulsively started oscillating vertical plate with radiation, chemical reaction and Hall current. The finite element method has been adopted to solve the governing equations of the flow. The effects of embedded parameters on primary and secondary fluid velocities, fluid temperature, fluid concentration, primary and secondary skin frictions, Nusselt number and Sherwood number are presented through the graphs and tables and then discussed. According to the best of authors' knowledge this work not yet received the attention of researchers though it has significant applications in the fields of science and engineering.

\section{Model Problem}

Consider the unsteady MHD flow of viscous incompressible electrically conducting, radiating and reacting fluid past an

impulsively started oscillating infinite vertical plate. A uniform magnetic field $\vec{B}$ of strength $B_{0}$ is applied in the direction perpendicular to the fluid flow. In the Cartesian co-ordinate system, the $x^{\prime}$-axis is taken along the plate in the vertically upward direction, the $y^{\prime}$-axis perpendicular to the direction of the plate and the $z^{\prime}-$ axis is normal to the $x^{\prime} y^{\prime}-$ plane. The physical model of the problem is shown in Fig.1. Initially, at time $t^{\prime} \leq 0$, the temperature of the fluid and the plate is $T_{\infty}^{\prime}$ and the concentration of the fluid is $C_{\infty}^{\prime}$. Subsequently, at time $t^{\prime}>0$, the plate starts oscillating in its own plane with frequency $\omega^{\prime}$, the temperature of the plate and the concentration of the fluid, respectively are raised to $T_{w}^{\prime}$ and $C_{w}^{\prime}$. It is assumed that the radiation heat flux in the $x^{\prime}-$ direction is negligible as compared to that in $y^{\prime}$-direction. As the plate is of infinite extent and electrically non-conducting, all the physical quantities, except the pressure, are functions of $y^{\prime}$ and $t^{\prime}$.

The generalized Ohm's law on taking Hall current into account Cowling (1957) is given by

$$
\vec{J}+\frac{\omega_{e} \tau_{e}}{B_{0}}(\vec{J} \times \vec{B})=\sigma(\vec{B}+\vec{q} \times \vec{B})
$$

where $\vec{q}, \vec{B}, \vec{E}, \vec{J}, \sigma, \omega_{e}$ and $\tau_{e}$ are respectively, velocity vector, magnetic field vector, electric field vector, current density vector, electric conductively, cyclotron frequency and electron collision time. The equation of continuity $\nabla \cdot \vec{q}=0 \quad$ gives $\quad v^{\prime}=0$ everywhere in the flow since there is no variation of the flow in $y^{\prime}$-direction, where $\vec{q}=\left(u^{\prime}, v^{\prime}, w^{\prime}\right)$ and $u^{\prime}, v^{\prime}, w^{\prime}$ are respectively, velocity components along the coordinate axes. 


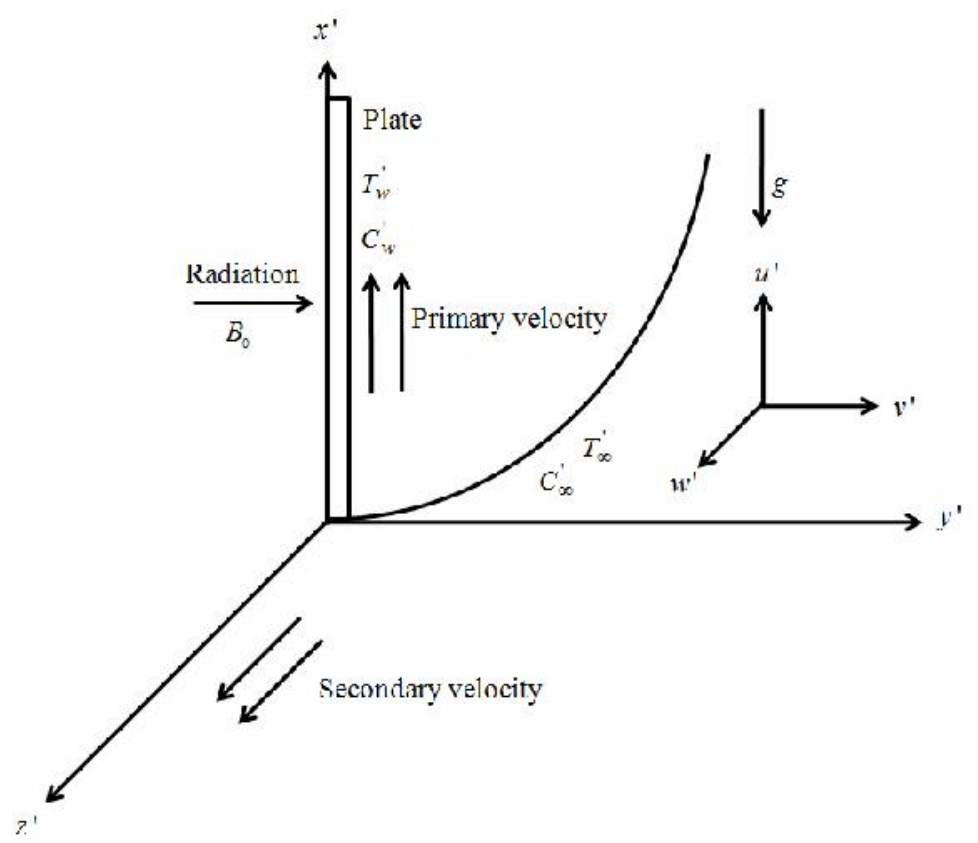

Figure 1. Physical model and coordinate system.

The magnetic Reynolds number is so small that the induced magnetic field produced by the fluid motion is neglected. The solenoid relation $\nabla \cdot \vec{B}=0$ for the magnetic field $\vec{B}=\left(B_{x^{\prime}}, B_{y^{\prime}}, B_{z^{\prime}}\right)$ gives $B_{y^{\prime}}=$ constant say $B_{0}$. i.e., $\vec{B}=\left(0, B_{0}, 0\right)$ everywhere in the flow. The conservation of electric current $\nabla . \vec{J}=0$ yields $j_{y^{\prime}}=$ constant, where $\vec{J}=\left(j_{x^{\prime}}, j_{y^{\prime}}, j_{z^{\prime}}\right)$. This constant is zero since $j_{y^{\prime}}=0$ at the plate which is electrically non-conducting. Hence, $j_{y^{\prime}}=0$ everywhere in the flow. In view of the above assumption, Eq. (1) yields

$$
\begin{aligned}
& j_{x^{\prime}}-m j_{y^{\prime}}=\sigma\left(E_{x^{\prime}}-w^{\prime} B_{0}\right) \\
& j_{z^{\prime}}+m j_{x^{\prime}}=\sigma\left(E_{z^{\prime}}+u^{\prime} B_{0}\right)
\end{aligned}
$$

where $m\left(=\omega_{e} \tau_{e}\right)$ is the Hall parameter which represents the ratio of electron-cyclotron frequency and the electron-atom collision frequency. Since the induced magnetic field is neglected, Maxwell equation $\nabla \times \vec{E}=-\frac{\partial \vec{H}}{\partial t}$ becomes $\nabla \times \vec{E}=0$ which gives $\frac{\partial E_{x^{\prime}}}{\partial y^{\prime}}=0$ and $\frac{\partial E_{z^{\prime}}}{\partial y^{\prime}}=0$. This implies that $E_{x^{\prime}}=$ constant and $E_{z^{\prime}}=$ constant everywhere in the flow and choose this constants equals to zero, i.e., $E_{x^{\prime}}=E_{z^{\prime}}=0$. Solving for $j_{x^{\prime}}$ and $j_{z^{\prime}}$ from Eqs. (2) and (3), on using $E_{x^{\prime}}=E_{z^{\prime}}=0$,

$$
\begin{aligned}
& j_{x^{\prime}}=\frac{\sigma B_{0}}{1+m^{2}}\left(m u^{\prime}-w^{\prime}\right) \\
& j_{z^{\prime}}=\frac{\sigma B_{0}}{1+m^{2}}\left(m w^{\prime}+u^{\prime}\right)
\end{aligned}
$$

Taking into consideration the assumptions made above, under the Boussinesq's approximation, and using Eqs. (4) and (5), the basic governing equations of the flow are derived as:

$$
\begin{aligned}
& \frac{\partial u^{\prime}}{\partial t^{\prime}}=v \frac{\partial^{2} u^{\prime}}{\partial y^{\prime 2}}-\frac{\sigma B_{0}^{2}}{\rho\left(1+m^{2}\right)}\left(u^{\prime}+m w^{\prime}\right)+g \beta\left(T^{\prime}-T_{\infty}^{\prime}\right)+g \beta^{*}\left(C^{\prime}-C_{\infty}^{\prime}\right) \\
& \frac{\partial w^{\prime}}{\partial t^{\prime}}=v \frac{\partial^{2} w^{\prime}}{\partial y^{\prime 2}}+\frac{\sigma B_{0}^{2}}{\rho\left(1+m^{2}\right)}\left(m u^{\prime}-w^{\prime}\right) \\
& \rho C_{p} \frac{\partial T^{\prime}}{\partial t^{\prime}}=k \frac{\partial^{2} T^{\prime}}{\partial y^{\prime 2}}-\frac{\partial q_{r}}{\partial y^{\prime}}
\end{aligned}
$$


$\frac{\partial C^{\prime}}{\partial t^{\prime}}=D \frac{\partial^{2} C^{\prime}}{\partial y^{\prime 2}}-\gamma^{\prime}\left(C^{\prime}-C_{\infty}^{\prime}\right)$

The initial and boundary conditions for the problem are:

$$
\begin{gathered}
t^{\prime} \leq 0 ; u^{\prime}=0, w^{\prime}=0, T^{\prime}=T_{\infty}{ }^{\prime}, C^{\prime}=C_{\infty}{ }^{\prime} \quad \text { for all } y^{\prime} \geq 0 \\
t^{\prime}>0 ; u^{\prime}=u_{0} \cos \omega^{\prime} t^{\prime}, w^{\prime}=0, T^{\prime}=T_{\infty}^{\prime}+\left(T_{w}{ }^{\prime}-T_{\infty}^{\prime}\right) \frac{t^{\prime} u_{0}^{2}}{v}, C^{\prime}=C_{w}^{\prime} \quad \text { at } y^{\prime}=0 \\
u^{\prime} \rightarrow 0, w^{\prime} \rightarrow 0, T^{\prime} \rightarrow T_{\infty}{ }^{\prime}, C^{\prime} \rightarrow C_{\infty}{ }^{\prime} \quad \text { as } y^{\prime} \rightarrow \infty
\end{gathered}
$$

The radiation heat flux $q_{r}$ under the Rosseland approximation Magyari and Pantokratoras (2011) expressed by

$$
q_{r}=-\frac{4 \sigma^{*}}{3 k^{*}} \frac{\partial T^{\prime 4}}{\partial y^{\prime}}
$$

where $\sigma^{*}$ is the Stefan-Boltzmann constant and $k^{*}$ is the mean absorption coefficient. It is assumed that temperature difference within the flow are sufficiently small, then Eq. (11) can be linearized by expanding $T^{\prime 4}$ into the Taylor series about $T_{\infty}^{\prime}$ which, after neglecting higher-order terms, takes the form:

$T^{\prime 4} \cong 4 T_{\infty}^{\prime 3} T^{\prime}-3 T_{\infty}^{\prime 4}$

In view of Eqs. (11) and (12), Eq. (8) reduces to

$$
\rho C_{p} \frac{\partial T^{\prime}}{\partial t^{\prime}}=k\left(1+\frac{16 \sigma^{*} T_{\infty}^{\prime 3}}{3 k k^{*}}\right) \frac{\partial^{2} T^{\prime}}{\partial y^{\prime 2}}
$$

Let us introduce the following non-dimensional parameters and quantities:

$$
\begin{aligned}
& u=\frac{u^{\prime}}{u_{0}}, y=\frac{y^{\prime} u_{0}}{v}, t=\frac{t^{\prime} u_{0}{ }^{2}}{v}, w=\frac{w^{\prime}}{u_{0}}, \omega=\frac{\omega^{\prime} v}{u_{0}^{2}}, S_{c}=\frac{v}{D}, P_{r}=\frac{\mu C_{p}}{k}, M=\frac{\sigma B_{0}{ }^{2} v}{\rho u_{0}^{2}}, \gamma=\frac{\gamma^{\prime} v}{u_{0}^{2}}, \\
& R=\frac{16 \sigma^{*} T_{\infty}^{\prime 3}}{3 k k^{*}}, \theta=\frac{\left(T^{\prime}-T_{\infty}^{\prime}\right)}{\left(T_{w}^{\prime}-T_{\infty}^{\prime}\right)}, \phi=\frac{\left(C^{\prime}-C_{\infty}^{\prime}\right)}{\left(C_{w}^{\prime}-C_{\infty}^{\prime}\right)}, G_{r}=\frac{g \beta v\left(T_{w}^{\prime}-T_{\infty}^{\prime}\right)}{u_{0}{ }^{3}}, G_{m}=\frac{g \beta v\left(C_{w}^{\prime}-C_{\infty}^{\prime}\right)}{u_{0}{ }^{3}}
\end{aligned}
$$

Using Eq. (14), into Eqs. (6), (7),(9) and (13), the following dimensionless governing equations of the flow are obtained:

$$
\begin{aligned}
& \frac{\partial u}{\partial t}=\frac{\partial^{2} u}{\partial y^{2}}-\frac{M}{\left(1+m^{2}\right)}(u+m w)+G_{r} \theta+G_{m} \phi \\
& \frac{\partial w}{\partial t}=\frac{\partial^{2} w}{\partial y^{2}}+\frac{M}{\left(1+m^{2}\right)}(m u-w) \\
& \frac{\partial \theta}{\partial t}=\frac{(1+R)}{P_{r}} \frac{\partial^{2} \theta}{\partial y^{2}} \\
& \frac{\partial \phi}{\partial t}=\frac{1}{S_{c}} \frac{\partial^{2} \phi}{\partial y^{2}}-\gamma \phi
\end{aligned}
$$

The initial and boundary conditions Eq. (10), in non-dimensional form become:

$$
\begin{aligned}
t \leq 0 ; u & =0, w=0, \theta=0, \phi=0 & & \text { for all } y \geq 0 \\
t>0 ; u & =\cos \omega t, w=0, \theta=t, \phi=1 & & \text { at } y=0 \\
u & \rightarrow 0, w \rightarrow 0, \theta \rightarrow 0, \phi \rightarrow 0 & & \text { as } y \rightarrow \infty
\end{aligned}
$$

\section{Numerical Procedure}

The dimensionless governing system of partial differential equations given in Eqs. (15) - (18), subject to initial and boundary conditions Eq, (19) are solved numerically for various values of parameters involved in the problem by using the finite element method. The finite element method is extremely efficient technique to solve ordinary and partial differential equations which occurs in wide range of engineering problems such as fluid mechanics, heat transfer, electrical systems, solid mechanics and chemical engineering, etc. Detailed discussion of the method is given in Zienkiewiez (1971), Reddy (1985) and Bathe (1996). 


\section{Variational Formulation}

The variational form associated with Eqs. (15) - (18), over a typical two nodded linear element $(e),\left(y_{j} \leq y \leq y_{k}\right)$ is given by

$$
\begin{aligned}
& \int_{y_{j}}^{y_{k}} w_{1}\left[\left(\frac{\partial u}{\partial t}\right)-\left(\frac{\partial^{2} u}{\partial y^{2}}\right)+\frac{M}{\left(1+m^{2}\right)}(u+m w)-\left(G_{r} \theta+G_{m} \phi\right)\right] d y=0 \\
& \int_{y_{j}}^{y_{k}} w_{2}\left[\left(\frac{\partial w}{\partial t}\right)-\left(\frac{\partial^{2} w}{\partial y^{2}}\right)+\frac{M}{\left(1+m^{2}\right)}(m u-w)\right] d y=0 \\
& \int_{y_{j}}^{y_{k}} w_{3}\left[\left(\frac{\partial \theta}{\partial t}\right)-\frac{(1+R)}{P_{r}}\left(\frac{\partial^{2} \theta}{\partial y^{2}}\right)\right] d y=0 \\
& \int_{y_{j}}^{y_{k}} w_{4}\left[\left(\frac{\partial \phi}{\partial t}\right)-\frac{1}{S_{c}}\left(\frac{\partial^{2} \phi}{\partial y^{2}}\right)+\gamma \phi\right] d y=0
\end{aligned}
$$

where $w_{1}, w_{2}, w_{3}$ and $w_{4}$ are arbitrary test functions and may be viewed as the variations in $u, w, \theta$ and $\phi$, respectively. After reducing the order of integration and non-linearity, the following equations are obtained:

$$
\begin{aligned}
& \int_{y_{j}}^{y_{k}}\left[\left(\frac{\partial w_{1}}{\partial y}\right)\left(\frac{\partial u}{\partial y}\right)+w_{1}\left(\frac{\partial u}{\partial t}\right)+\frac{M}{\left(1+m^{2}\right)} w_{1}(u+m w)\right] d y-\left[w_{1}\left(\frac{\partial u}{\partial y}\right)\right]_{y_{j}}^{y_{k}}=0 \\
& \int_{y_{j}}^{y_{k}}\left[\left(\frac{\partial w_{2}}{\partial y}\right)\left(\frac{\partial w}{\partial y}\right)+w_{2}\left(\frac{\partial w}{\partial t}\right)+\frac{M}{\left(1+m^{2}\right)} w_{2}(m u-w)\right] d y-\left[w_{2}\left(\frac{\partial w}{\partial y}\right)\right]_{y_{j}}^{y_{k}}=0 \\
& \int_{y_{j}}^{y_{k}}\left[\frac{(1+R)}{P_{r}}\left(\frac{\partial w_{3}}{\partial y}\right)\left(\frac{\partial \theta}{\partial y}\right)+w_{3}\left(\frac{\partial \theta}{\partial t}\right)\right] d y-\frac{(1+R)}{P_{r}}\left[w_{3}\left(\frac{\partial \theta}{\partial y}\right)\right]_{y_{j}}^{y_{k}}=0 \\
& y_{k}\left[\frac{1}{S_{c}}\left(\frac{\partial w_{4}}{\partial y}\right)\left(\frac{\partial \phi}{\partial y}\right)+w_{4}\left(\frac{\partial \phi}{\partial t}\right)-(\gamma \phi) w_{4}\right] d y-\frac{1}{S_{c}}\left[w_{4}\left(\frac{\partial \phi}{\partial y}\right)\right]_{y_{j}}^{y_{k}}=0
\end{aligned}
$$

\section{Finite Element Formulation}

The finite element model may be obtained from Eqs. (24) - (27) by substituting appropriate finite element approximations over the element $(e),\left(y_{j} \leq y \leq y_{k}\right)$ of the form:

$$
u^{(e)}=\sum_{j=1}^{2} u_{j} \psi_{j}, w^{(e)}=\sum_{j=1}^{2} w_{j} \psi_{k}, \theta^{(e)}=\sum_{j=1}^{2} \theta_{j} \psi_{j}, \phi^{(e)}=\sum_{j=1}^{2} \phi_{j} \psi_{j}
$$

with $w_{1}=w_{2}=w_{3}=w_{4}=\psi_{j}(j=1,2)$ where $u_{j}, w_{j}, \theta_{j}$ and $\phi_{j}$ are, respectively, primary fluid velocity, secondary fluid velocity, fluid temperature and fluid concentration at $j^{\text {th }}$ node of the element $(e)$ and $\psi_{j}$ 's are the shape functions for a typical element $(e),\left(y_{j} \leq y \leq y_{k}\right)$ and are taken as:

$\psi_{j}=\frac{y_{k}-y}{y_{k}-y_{j}}$ and $\psi_{k}=\frac{y-y_{j}}{y_{k}-y_{j}}, \quad\left(y_{j} \leq y \leq y_{k}\right)$

Using Eqs. (28) and (29) into Eqs. (24) to (27), after assembly of all the element equations by inter-element connectivity conditions and using Eq. (19), we obtain the following tri-diagonal system of equations:

$A u=A^{\prime} ; B w=B^{\prime} ; C \theta=C^{\prime} ; D \phi=D^{\prime}$ 
where $u, w, \theta, \phi$ and $A^{\prime}, B^{\prime}, C^{\prime}, D^{\prime}$ are column matrices having $n$-components, $u_{i}^{j+1}, w_{i}^{j+1}, \theta_{i}^{j+1}, \phi_{i}^{j+1}$ and $u_{i}^{j}, w_{i}^{j}, \theta_{i}^{j}, \phi_{i}^{j}$ respectively, and $A, B, C$ and $D$ are diagonal matrices of order $-n$ whose elements are given by

$$
\begin{array}{ll}
a_{i-1, i}=1-3 r+\frac{1}{2} r\left(\frac{M}{1+m^{2}}\right) h^{2} ; b_{i-1, i}=1-3 r+\frac{1}{2} r\left(\frac{M}{1+m^{2}}\right) h^{2} ; c_{i-1, i}=\left(\frac{P_{r}}{1+R}\right)-3 r ; d_{i-1, i}=S_{c}-3 r+\frac{1}{2} r S_{c} \gamma h^{2} & \text { at } i=2(1) n \\
a_{i, i}=4+6 r+\frac{1}{2} r\left(\frac{M}{1+m^{2}}\right) h^{2} ; b_{i, i}=4+6 r+\frac{1}{2} r\left(\frac{M}{1+m^{2}}\right) h^{2} ; c_{i, i}=4\left(\frac{P_{r}}{1+R}\right)+6 r ; d_{i, i}=4 S_{c}+6 r+2 r S_{c} \gamma h^{2} & \text { at } i=1(1) n \\
a_{i, i-1}=1-3 r+\frac{1}{2} r\left(\frac{M}{1+m^{2}}\right) h^{2} ; b_{i, i-1}=1-3 r+\frac{1}{2} r\left(\frac{M}{1+m^{2}}\right) h^{2} ; c_{i, i-1}=\left(\frac{P_{r}}{1+R}\right)-3 r ; d_{i, i-1}=S_{c}-3 r+\frac{1}{2} r S_{c} \gamma h^{2} & \text { at } i=2(1) n
\end{array}
$$

Here, $r=k / h^{2}$ and $h, k$ are mesh sizes along $y, t$-directions, respectively. For computational purpose the region of integration is considered as a rectangle with sides $t_{\max }=3$ and $y_{\max }=5$ where $y_{\max }$ corresponds to $(y=\infty)$, which lies very well outside the momentum, thermal and the concentration boundary layers. The Gauss elimination scheme is employed to solve the system of equations given in Eq. (30) by maintaining accuracy 0.00005. Numerical solutions for the primary and secondary fluid velocities $u$ and $w$, fluid temperature $\theta$ and fluid concentration $\phi$, a code was set up in $C++$ language. The mesh size $h=0.05$ and $k=0.0025$ was selected after comparing the results when the code was run with smaller values of $k=0.001,0.0009$ where it was noticed that the difference between these values is less than half a unity in the fourth decimal place. Also, it was found that $\left|f_{i}^{j+1}-f_{i}^{j}\right|<10^{-5},(f=u, w, \theta, \phi)$ at all nodal points. This designates that the method is stable. To judge the convergence of the method, computations are carried out by making small changes in the values of $h$ and $k$, no significant change was noticed in the values of $u, w, \theta$ and $\phi$. Hence, we conclude that finite element method is stable and convergent.

The dimensionless primary and secondary skin frictions are given by

$$
\tau_{x}=\left.\frac{\partial u}{\partial y}\right|_{y=0} \text { and } \quad \tau_{z}=\left.\frac{\partial w}{\partial y}\right|_{y=0}
$$

The dimensionless Nusselt and Sherwood numbers are given by

$$
N u=\left.\frac{\partial \theta}{\partial y}\right|_{y=0} \text { and } \quad S h=\left.\frac{\partial \phi}{\partial y}\right|_{y=0}
$$

\section{Results Validation}

To validate the present results, a comparison has been made by considering primary and secondary skin frictions $\tau_{x}$ and $\tau_{z}$ in the absence of radiation and chemical reaction with the results of Rajput and Kanaujia (2016) obtained by Laplace transform technique shown in Table 1 and 2. An excellent agreement is noticed in this comparison which validates the present numerical scheme. This justifies the correctness of the results presented in the manuscript.

Table 1. Comparison of primary skin friction between the present results and results by Rajput and Kanaujia (2016) in the absence of radiation and chemical reaction, i.e., $R=0$ and $\gamma=0$.

\begin{tabular}{|c|c|c|c|c|c|c|c|c|c|}
\hline$G_{r}$ & $G_{m}$ & $P_{r}$ & $S_{c}$ & $M$ & $m$ & $\omega t$ & & \multirow{2}{*}{$\begin{array}{c}\text { Present } \\
\text { results by } \\
\text { (degree) }\end{array}$} & $\begin{array}{c}\text { Results by Rajput } \\
\text { and Kanaujia } \\
\tau_{x}\end{array}$ \\
\hline
\end{tabular}




\begin{tabular}{|l|l|l|l|l|l|l|l|l|l|}
\hline 10.0 & 10.0 & 0.71 & 2.01 & 2.0 & 0.5 & 30 & 0.2 & 1.1780 & 1.1778 \\
20.0 & 10.0 & 0.71 & 2.01 & 2.0 & 0.5 & 30 & 0.2 & 1.5309 & 1.5307 \\
10.0 & 20.0 & 0.71 & 2.01 & 2.0 & 0.5 & 30 & 0.2 & 3.1769 & 3.1768 \\
10.0 & 10.0 & 7.00 & 2.01 & 2.0 & 0.5 & 30 & 0.2 & 1.0070 & 1.0063 \\
10.0 & 10.0 & 0.71 & 5.00 & 2.0 & 0.5 & 30 & 0.2 & 0.6887 & 0.6887 \\
10.0 & 10.0 & 0.71 & 2.01 & 3.0 & 0.5 & 30 & 0.2 & 0.9621 & 0.9621 \\
10.0 & 10.0 & 0.71 & 2.01 & 2.0 & 1.0 & 30 & 0.2 & 1.3388 & 1.3387 \\
10.0 & 10.0 & 0.71 & 2.01 & 2.0 & 0.5 & 45 & 0.2 & 1.7315 & 1.7314 \\
10.0 & 10.0 & 0.71 & 2.01 & 2.0 & 0.5 & 30 & 0.25 & 1.5749 & 1.5744 \\
\hline
\end{tabular}

Table 2. Comparison of secondary skin friction between the present results and results by Rajput and Kanaujia (2016) in the absence of radiation and chemical reaction, i.e., $R=0$ and $\gamma=0$.

\begin{tabular}{|c|c|c|c|c|c|c|c|c|c|}
\hline$G_{r}$ & $G_{m}$ & $P_{r}$ & $S_{c}$ & $M$ & $m$ & $\begin{array}{c}\omega t \\
(\text { degree })\end{array}$ & $t$ & $\begin{array}{c}\text { Present } \\
\text { results by } \\
\text { FEM } \\
\tau_{z}\end{array}$ & $\begin{array}{c}\text { Results by Rajput } \\
\text { and Kanaujia } \\
(2016) \\
\tau_{z}\end{array}$ \\
\hline 10.0 & 10.0 & 0.71 & 2.01 & 2.0 & 0.5 & 30 & 0.2 & 0.2149 & 0.2150 \\
20.0 & 10.0 & 0.71 & 2.01 & 2.0 & 0.5 & 30 & 0.2 & 0.2210 & 0.2208 \\
10.0 & 20.0 & 0.71 & 2.01 & 2.0 & 0.5 & 30 & 0.2 & 0.2560 & 0.2560 \\
10.0 & 10.0 & 7.00 & 2.01 & 2.0 & 0.5 & 30 & 0.2 & 0.2108 & 0.2107 \\
10.0 & 10.0 & 0.71 & 5.00 & 2.0 & 0.5 & 30 & 0.2 & 0.1975 & 0.1972 \\
10.0 & 10.0 & 0.71 & 2.01 & 3.0 & 0.5 & 30 & 0.2 & 0.3065 & 0.3062 \\
10.0 & 10.0 & 0.71 & 2.01 & 2.0 & 1.0 & 30 & 0.2 & 0.2790 & 0.2795 \\
10.0 & 10.0 & 0.71 & 2.01 & 2.0 & 0.5 & 45 & 0.2 & 0.1988 & 0.1983 \\
10.0 & 10.0 & 0.71 & 2.01 & 2.0 & 0.5 & 30 & 0.25 & 0.2490 & 0.2489 \\
\hline
\end{tabular}

\section{Graphical results and discussion}

In order to determine the effect of physical parameters on the flow fields such as magnetic parameter $M$, Hall parameter $m$, thermal Grashof number $G_{r}$, mass Grashof number $G_{m}$, Prandtl number $P_{r}$, Schmidt number $S_{c}$, radiation parameter $R$, chemical reaction parameter $\gamma$, phase angle $\omega t$ and time $t$, numerical computations have been carried out for primary velocity $u$, secondary velocity $w$, temperature $\theta$, concentration $\phi$, primary skin friction $\tau_{x}$, secondary skin friction $\tau_{z}$, Nusselt number $N_{u}$ and Sherwood number $S_{h}$. These obtained numerical results have been presented through the graphs and Tables and then discussed.

Figure 2 illustrate the effects of thermal Grashof number $G_{r}$ on the primary and secondary velocities. The thermal Grashof number $G_{r}$ signifies the ratio of thermal buoyancy force to viscous hydrodynamics force. As increase in $G_{r}$ tends to decrease drag forces and hence, fluid velocity increases. This implies that increase in the thermal Grashof number tends to accelerate fluid velocity in both primary and secondary flow directions. Here, the positive values of thermal Grashof number correspond to cooling of the plate. Figure 3 shows the effects of mass Grashof number $G_{m}$ on the primary and secondary velocities. The mass Grashof number $G_{m}$ signifies the ratio of species buoyancy force to the viscous hydrodynamic force. As mass Grashof number increases, the viscous hydrodynamic force decreases as a result momentum of the fluid increases. This implies that fluid velocity in both primary and secondary flow directions tends to increase with increasing $G_{m}$. Also, it is noticed that velocity components suddenly raise near to the plate and after this velocity components asymptotically decreases to zero as $y \rightarrow \infty$. Figure 4 represents the effect of magnetic parameter $M$ on the primary and secondary velocities. The application of a magnetic field perpendicular to the flow direction of an electrically conducting fluid, it experiences an electric field and produces current perpendicular to both magnetic field and flow direction. The product of electric field and magnetic field creates a force which is known as Lorentz force. The direction of the Lorentz force is always opposite to the direction of the flow in the absence of applied electric field which opposes the fluid velocity. This implies that increase in magnetic parameter tends to retard the fluid velocity in the primary flow direction whereas it tends to enhance the fluid velocity in the secondary flow direction. This tendency of the magnetic field is clearly supported by the physical reality. The effects of the Hall parameter $m$ on the primary and secondary velocities are depicted in Figure 5. It is observed that an increase in the Hall parameter tends to accelerate the fluid velocity in both primary and secondary 
flow directions. This situation clearly supports the fact that Hall current induces a cross-flow in the boundary layer. Figure 6 displays the effect of Prandtl number $P_{r}$ on the primary and secondary velocities. As increase in $P_{r}$ correspond to stronger momentum diffusivity and weaker thermal diffusivity. This implies that increase in the Prandtl number tends to decelerate fluid velocity in both primary and secondary flow direction. The influence of radiation parameter $R$ on the primary and secondary velocities is shown in Figure 7. It is seen that both primary and secondary velocities enhance with increasing radiation parameter. This is consistent with the definition of $R$. Figure 8 shows the effects of Schmidt number $S_{c}$ on the primary and secondary velocities. As $S_{c}$ increases fluid velocity in both primary and secondary flow directions is expected to reduce since increasingly momentum is diffused at a lower rate than species. The effects of chemical reaction parameter $\gamma$ on the primary and secondary velocities are presented in Figure 9. It is observed that an increase in $\gamma$ tends to decrease the fluid velocity in both primary and secondary flow direction. Figure 10 demonstrate the effects of phase angle $\omega t$ on the primary and secondary velocities. It can be seen that increase in the phase angle tends to decelerate the fluid velocity in both primary and secondary flow directions. Physically, as $\omega t$ increase buoyancy force tends to decrease and hence, decrease the fluid momentum. The influence of time parameter $t$ on the primary and secondary velocities is presented in Figure 11. As time progresses, fluid velocity in both primary and secondary flow directions are getting accelerated due to increasing buoyancy effects.

Figure 12 depicts the effect of Prandtl number $P_{r}$ on the fluid temperature. Prandtl number signifies the ratio of momentum to thermal diffusivities. As $P_{r}$ increases thermal boundary layer thickness decreases. This implies that increase in Prandtl number tends to decrease the fluid temperature across the boundary layer. The effects of radiation parameter $R$ on the fluid temperature are shown in Figure13. It is observed that increase in the radiation parameter tends to enhance the fluid temperature in the boundary layer. As time progress, the fluid temperature $\theta$ increases as seen in Figure 14. The effects of Schmidt number $S_{c}$ on the fluid concentration $\phi$ are presented in Figure 15. To be more realistic, the values of Schmidt number are chosen to represent the diffusing chemical species of most common interest like hydrogen $\left(S_{c}=0.23\right)$, water-vapor $\left(S_{c}=0.64\right)$, sulfur dioxide $\left(S_{c}=1.20\right)$ and naphthalene $\left(S_{c}=2.23\right)$. It is clear that an increase in Schmidt number tends to decrease the fluid concentration. Physically, increase in $S_{c}$ decrease molecular diffusivity which results in a decrease of concentration boundary layer. Figure 16 displays the effect of chemical reaction parameter $\gamma$ on the fluid concentration. It is seen that increase in $\gamma$ tends to decrease concentration of species in the boundary layer since large values of $\gamma$ reduce the solutal boundary layer thickness and increase the mass transfer. The variation of fluid concentration $\phi$ with progression of time $t$ is depicted in Figure 17. It is noticed that fluid concentration increases with increasing time $t$.

The numerical values of primary skin friction $\tau_{x}$, secondary skin friction $\tau_{z}$, Nusselt number $N_{u}$ and Sherwood number $S_{h}$ for variations in $G_{r}, G_{m}, P_{r}, S_{c}, M, m, R, \gamma, \omega t$ and $t$ are presented in Tables 3 to 5 , respectively. Table 3 reveals that an increase in $G_{r}, G_{m}, m, R$ and $t$ tends to increase both primary and secondary skin frictions whereas an increase in $P_{r}, S_{c}$ and $\gamma$ tends to decrease both primary and secondary skin frictions. Magnetic parameter $M$ tends to decelerate primary skin friction and accelerate secondary skin friction whereas phase angle has opposite effect. Table 4 shows that an increase in $P_{r}$ tends increase Nusselt number whereas it decreases with increasing $R$ and $t$. It is clearly seen from Table 5, that an increase in $S_{c}$ and $\gamma$ tends to increase Sherwood number whereas it decreases with increasing $t$.

\section{Figures and Tables}
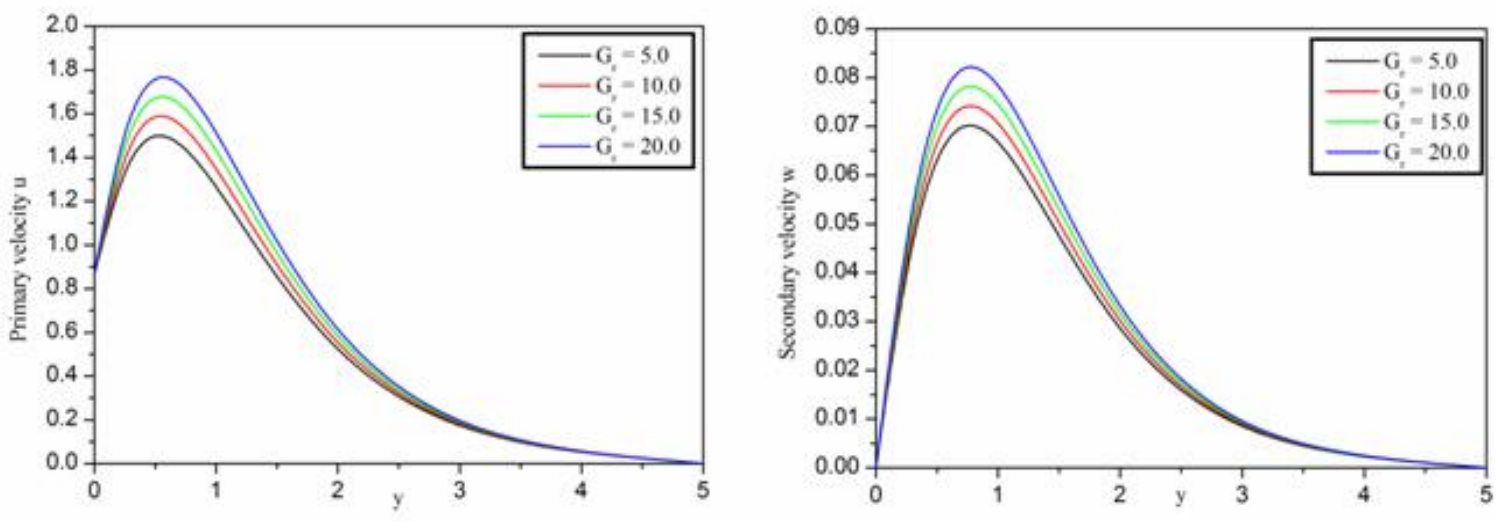

Figure 2. Primary and secondary velocities for varying $G_{r}$ when $G_{m}=10.0, M=1.0, m=0.5, P_{r}=0.71, R=1.0$, 


$$
S_{c}=0.22, \gamma=0.5, \omega t=30^{0}, t=0.2 \text {. }
$$
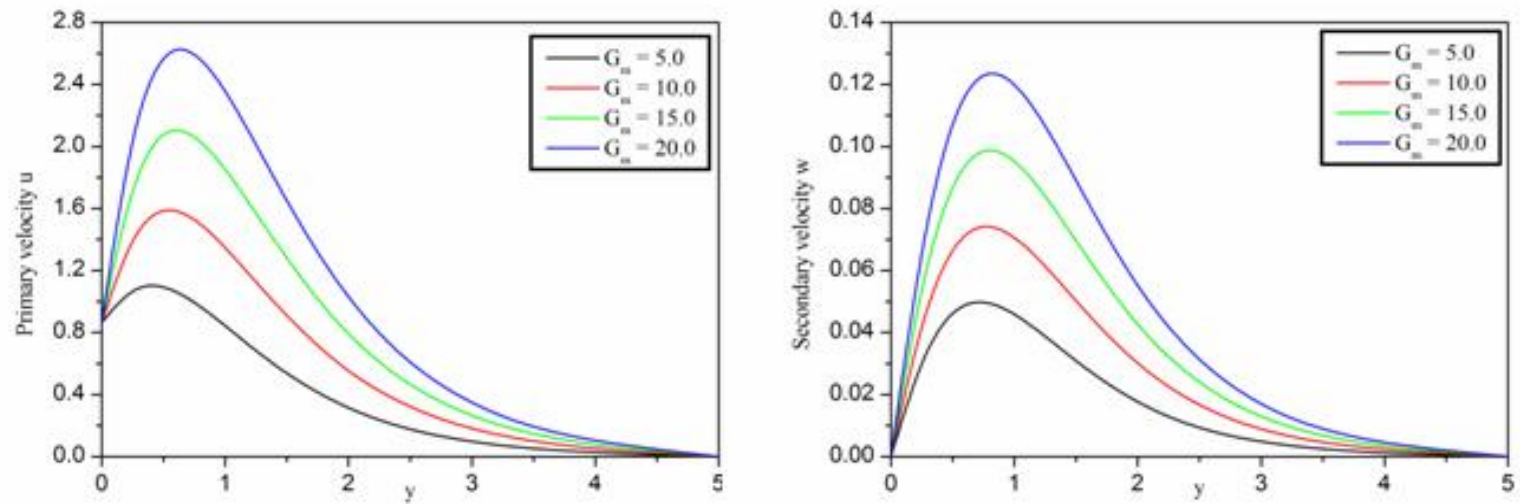

Figure 3. Primary and secondary velocities for varying $G_{m}$ when $G_{r}=10.0, M=1.0, m=0.5, P_{r}=0.71, R=1.0$,

$$
S_{c}=0.22, \gamma=0.5, \omega t=30^{0}, t=0.2 \text {. }
$$
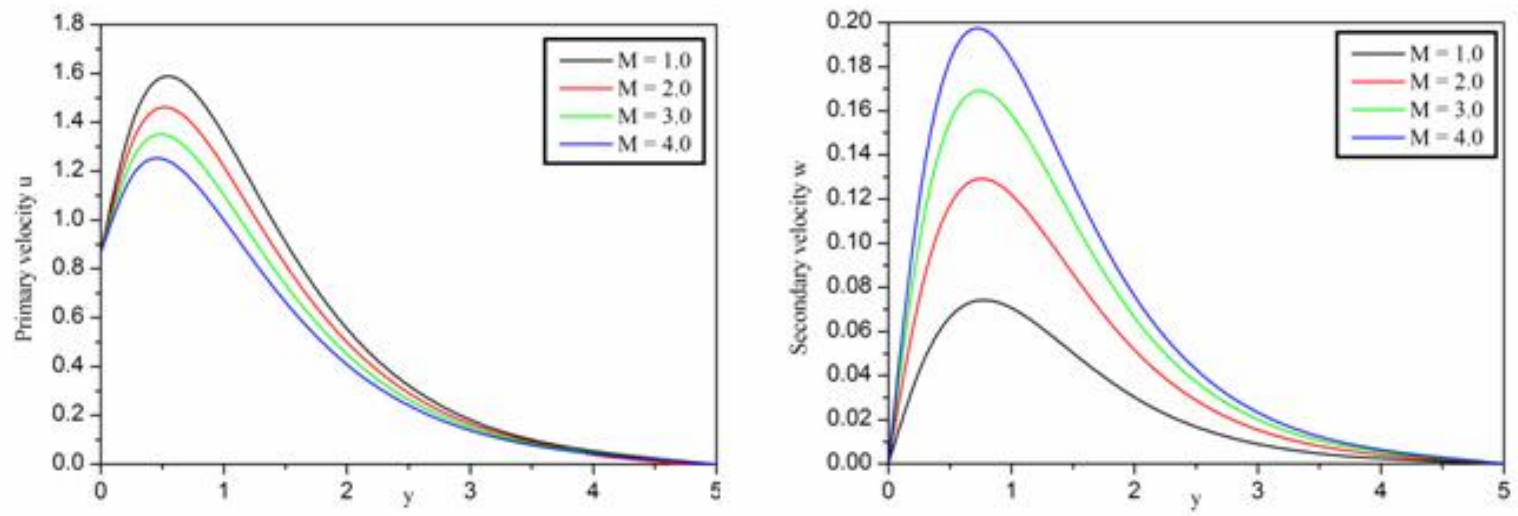

Figure 4. Primary and secondary velocities for varying $M$ when $G_{r}=10.0, G_{m}=10.0, m=0.5, P_{r}=0.71, R=1.0$,

$$
S_{c}=0.22, \gamma=0.5, \omega t=30^{\circ}, t=0.2 \text {. }
$$
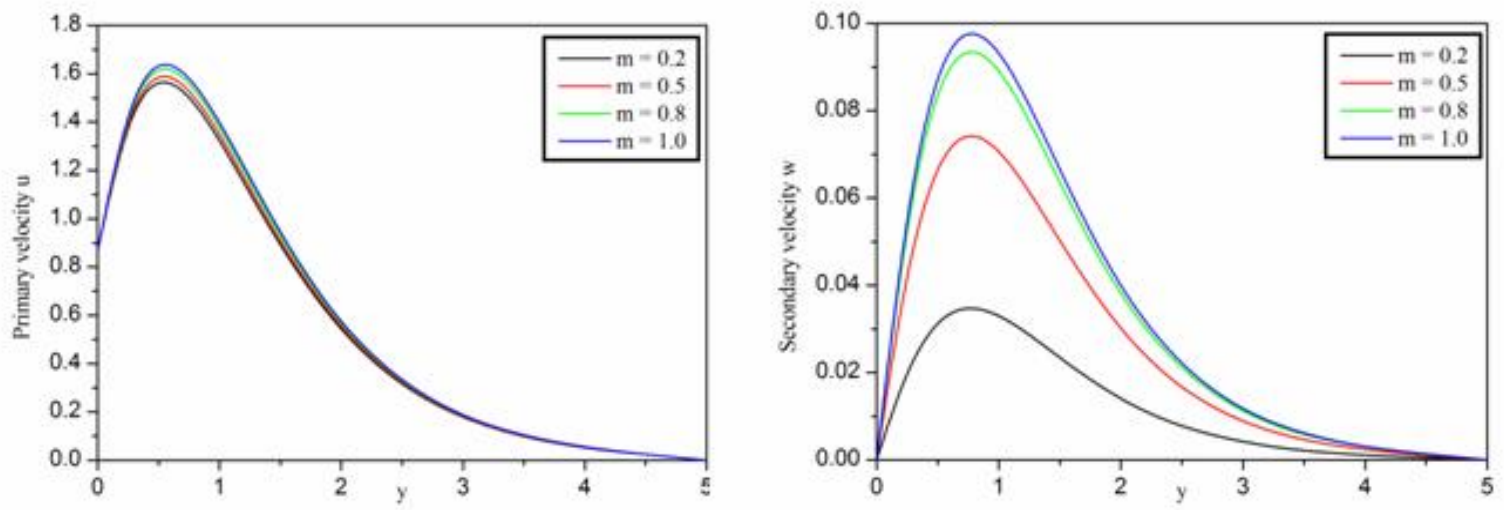

Figure 5. Primary and secondary velocities for varying $m$ when $G_{r}=10.0, G_{m}=10.0, M=1.0, P_{r}=0.71, R=1.0$,

$$
S_{c}=0.22, \gamma=0.5, \omega t=30^{0}, t=0.2 \text {. }
$$



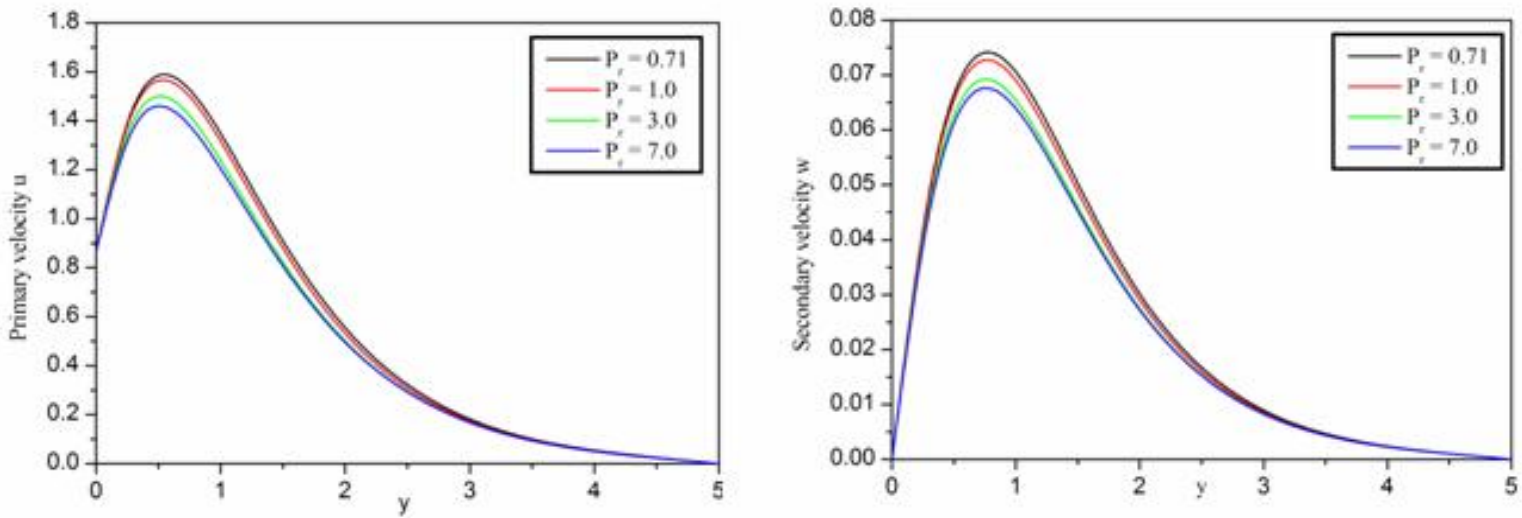

Figure 6. Primary and secondary velocities for varying $P_{r}$ when $G_{r}=10.0, G_{m}=10.0, M=1.0, m=0.5, R=1.0$,

$$
S_{c}=0.22, \gamma=0.5, \omega t=30^{0}, t=0.2 \text {. }
$$
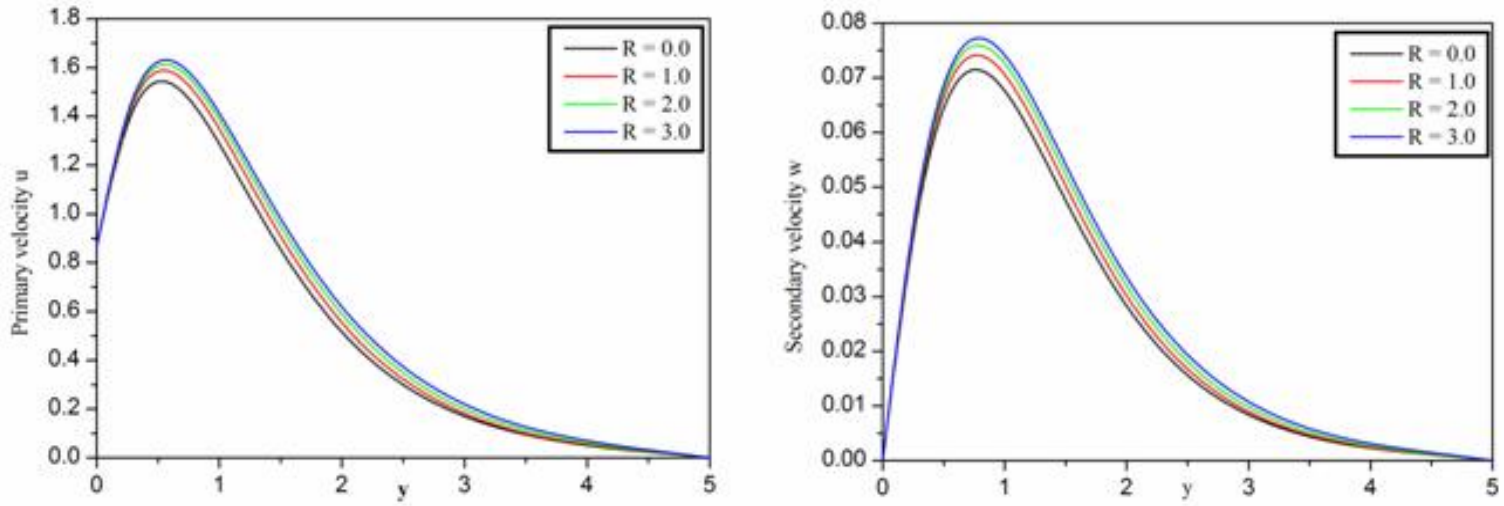

Figure 7. Primary and secondary velocities for varying $R$ when $G_{r}=10.0, G_{m}=10.0, M=1.0, m=0.5, P_{r}=0.71$,

$$
S_{c}=0.22, \gamma=0.5, \omega t=30^{\circ}, t=0.2 \text {. }
$$
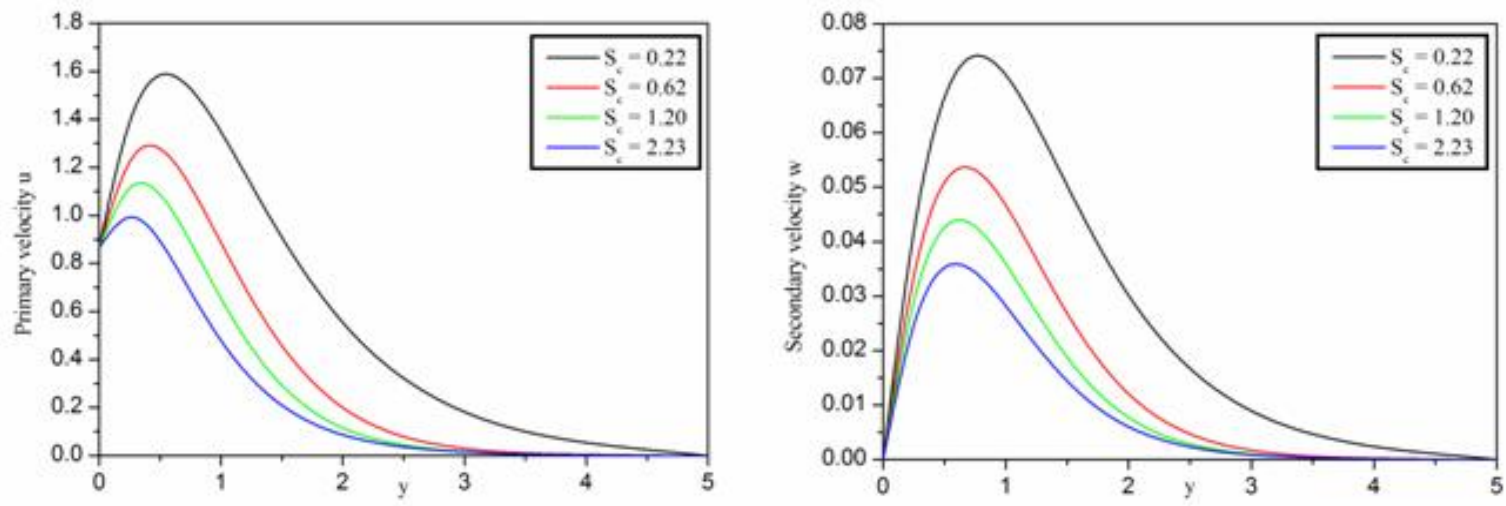

Figure 8. Primary and secondary velocities for varying $S_{c}$ when $G_{r}=10.0, G_{m}=10.0, M=1.0, m=0.5, P_{r}=0.71$,

$$
R=1.0, \gamma=0.5, \omega t=30^{\circ}, t=0.2 \text {. }
$$



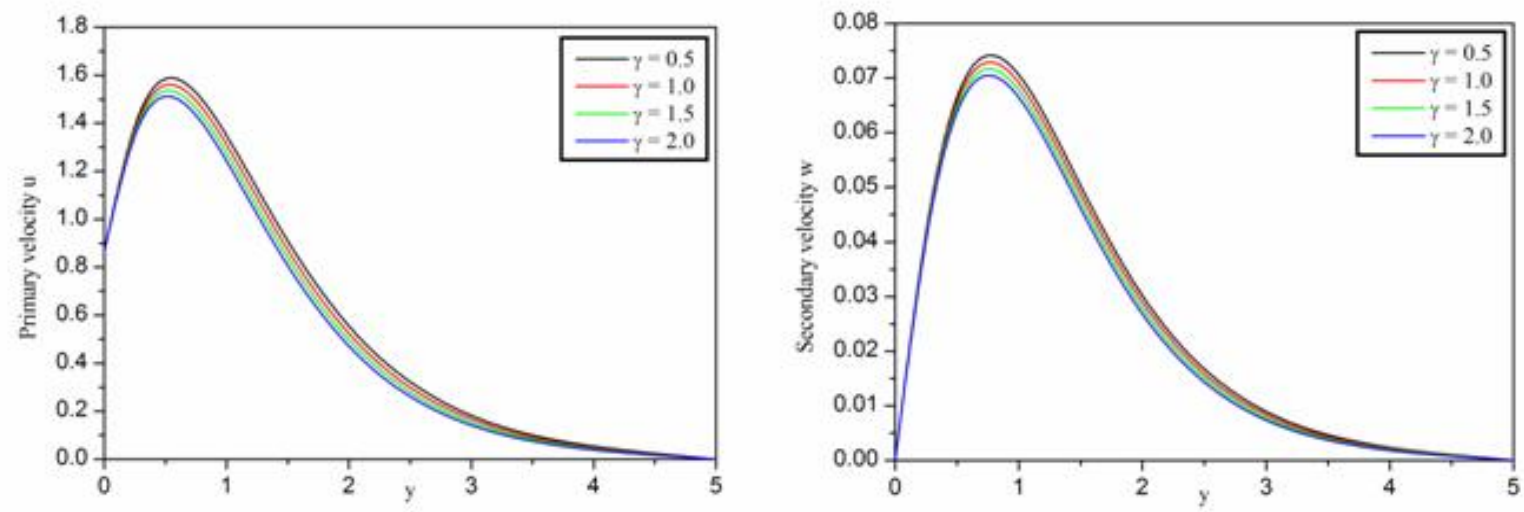

Figure 9. Primary and secondary velocities for varying $\gamma$ when $G_{r}=10.0, G_{m}=10.0, M=1.0, m=0.5, P_{r}=0.71$,

$$
R=1.0, S_{c}=0.22, \omega t=30^{\circ}, t=0.2 .
$$
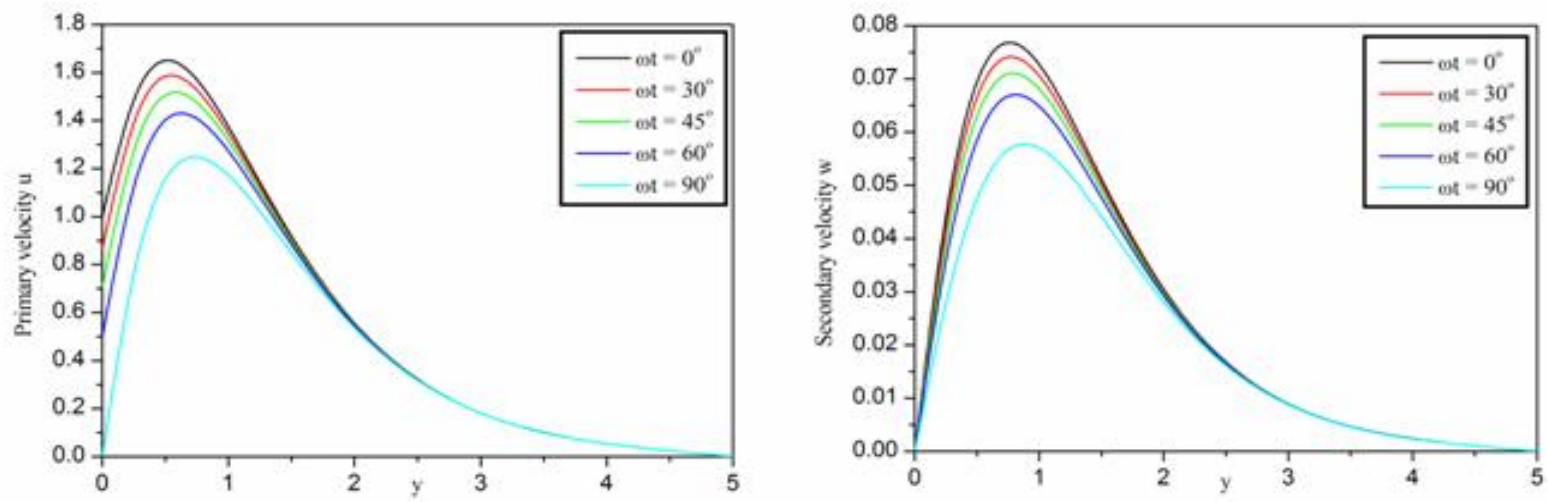

Figure 10. Primary and secondary velocities for varying $\omega t$ when $G_{r}=10.0, G_{m}=10.0, M=1.0, m=0.5, P_{r}=0.71$,

$$
R=1.0, S_{c}=0.22, \gamma=0.5, t=0.2 .
$$
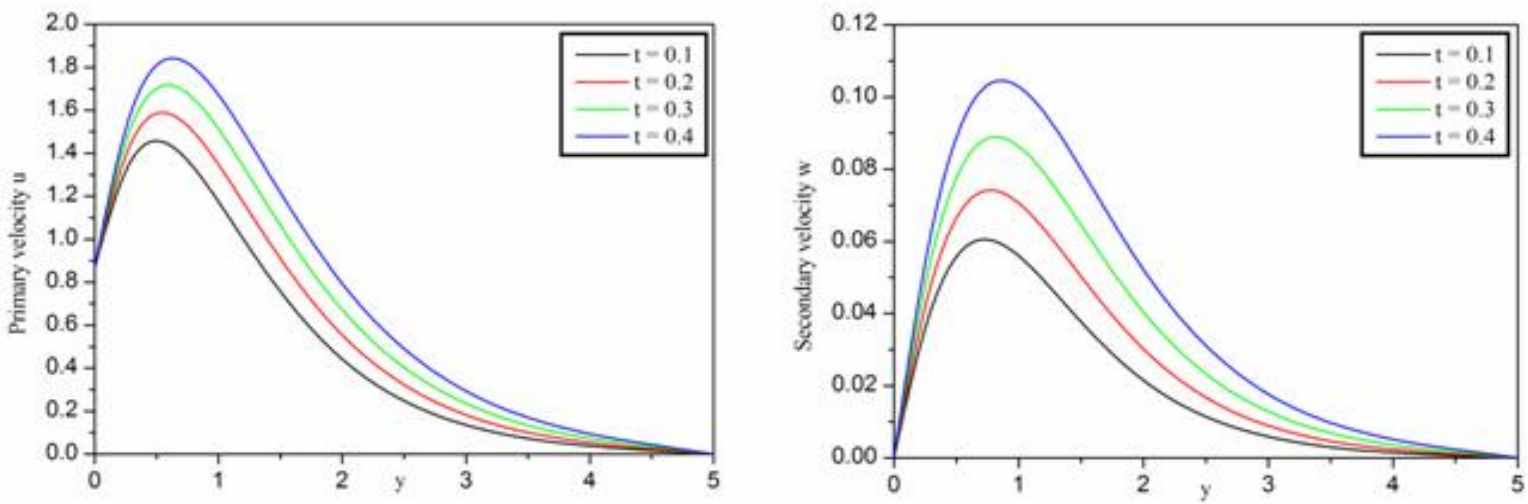

Figure 11. Primary and secondary velocities for varying $t$ when $G_{r}=10.0, G_{m}=10.0, M=1.0, m=0.5, P_{r}=0.71$,

$$
R=1.0, S_{c}=0.22, \gamma=0.5, \omega t=30^{\circ} .
$$




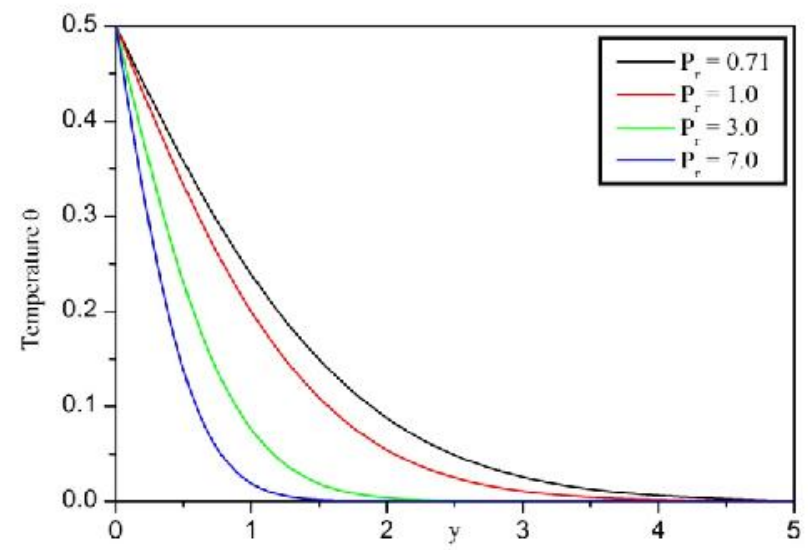

Figure 12. Temperature for varying Prandtl number $P_{r}$ when $R=1.0$ at $t=0.2$.

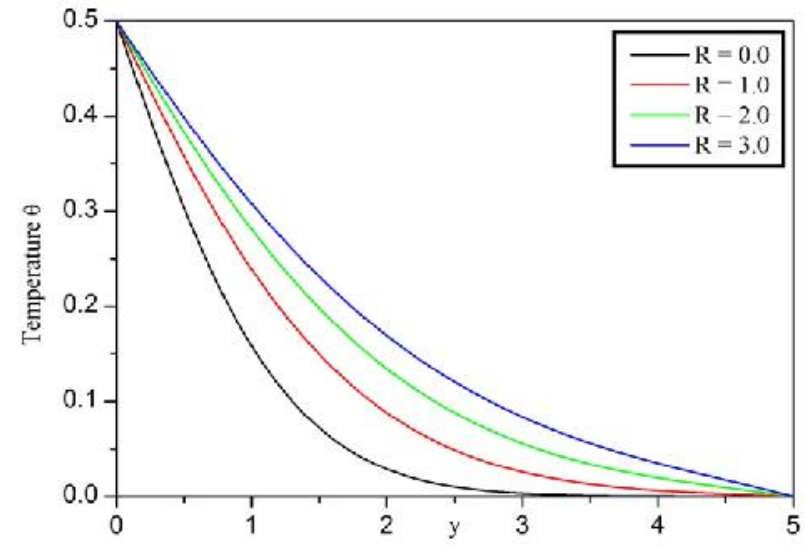

Figure 13. Temperature for varying radiation parameter $R$ when $P_{r}=0.71$ at $t=0.2$.

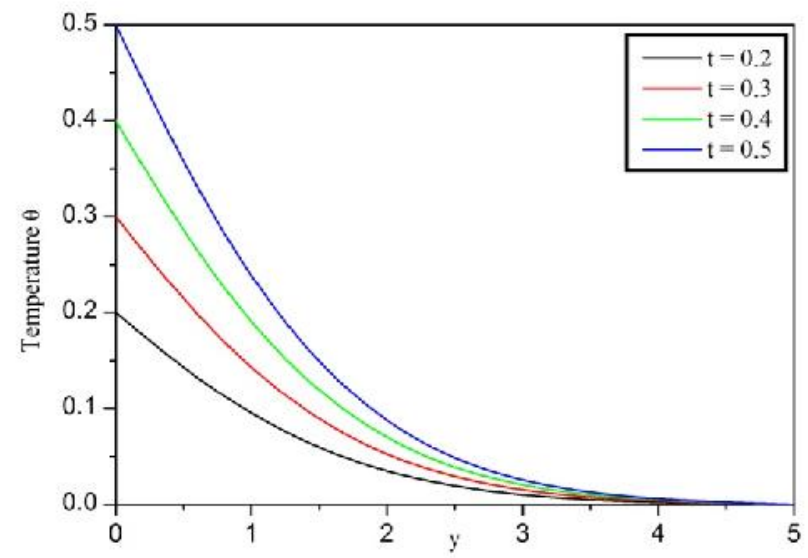

Figure 14. Temperature for varying time $t$ when $P_{r}=0.71$ and $R=1.0$. 


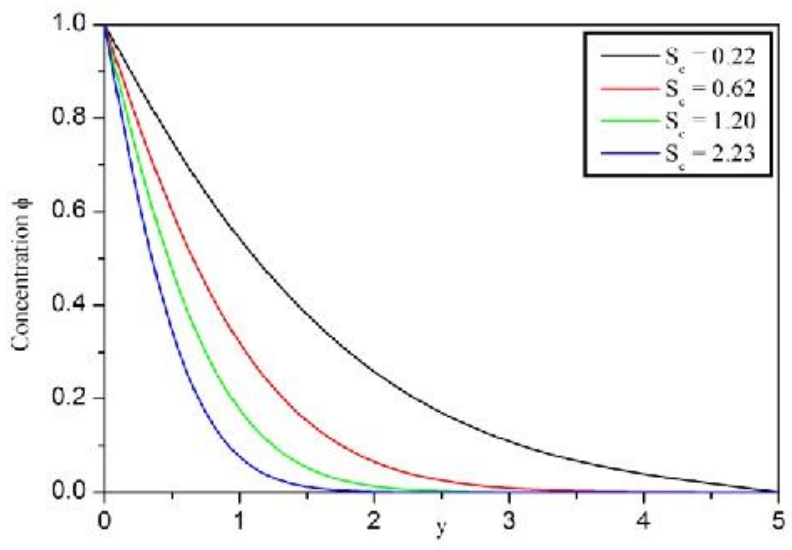

Figure 15. Concentration for varying Schmidt number $S_{c}$ when $\gamma=0.5$ at $t=0.2$.

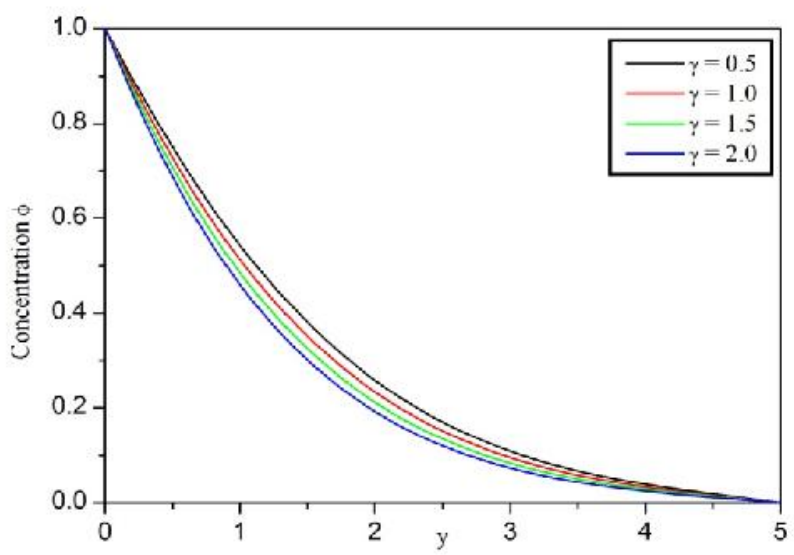

Figure 16. Concentration for varying chemical reaction parameter $\gamma$ when $S_{c}=0.22$ at $t=0.2$.

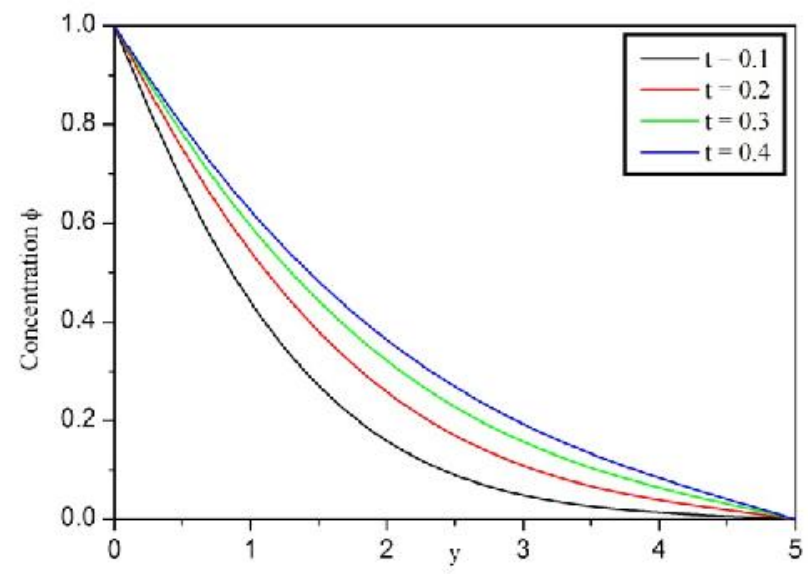

Figure 17. Concentration for varying time $t$ when $S_{c}=0.22$ and $\gamma=0.5$. 
Table 3. Numerical values of primary and secondary skin frictions

\begin{tabular}{|c|c|c|c|c|c|c|c|c|c|c|c|}
\hline$G_{r}$ & $G_{m}$ & $P_{r}$ & $S_{c}$ & $M$ & $m$ & $R$ & $\gamma$ & $\begin{array}{c}\omega t \\
(\text { Degree })\end{array}$ & $t$ & $\tau_{x}$ & $\tau_{z}$ \\
\hline 10.0 & 10.0 & 0.71 & 0.22 & 2.0 & 0.5 & 1.0 & 0.5 & 30 & 0.2 & 1.187770 & 0.182128 \\
20.0 & 10.0 & 0.71 & 0.22 & 2.0 & 0.5 & 1.0 & 0.5 & 30 & 0.2 & 1.448654 & 0.199436 \\
10.0 & 20.0 & 0.71 & 0.22 & 2.0 & 0.5 & 1.0 & 0.5 & 30 & 0.2 & 2.611304 & 0.282328 \\
10.0 & 10.0 & 7.00 & 0.22 & 2.0 & 0.5 & 1.0 & 0.5 & 30 & 0.2 & 1.034078 & 0.169832 \\
10.0 & 10.0 & 0.71 & 0.62 & 2.0 & 0.5 & 1.0 & 0.5 & 30 & 0.2 & 0.847760 & 0.148496 \\
10.0 & 10.0 & 0.71 & 0.22 & 3.0 & 0.5 & 1.0 & 0.5 & 30 & 0.2 & 0.866006 & 0.438436 \\
10.0 & 10.0 & 0.71 & 0.22 & 2.0 & 1.0 & 1.0 & 0.5 & 30 & 0.2 & 1.251342 & 0.237312 \\
10.0 & 10.0 & 0.71 & 0.22 & 2.0 & 0.5 & 2.0 & 0.5 & 30 & 0.2 & 1.213240 & 0.184796 \\
10.0 & 10.0 & 0.71 & 0.22 & 2.0 & 0.5 & 1.0 & 1.0 & 30 & 0.2 & 1.158930 & 0.180124 \\
10.0 & 10.0 & 0.71 & 0.22 & 2.0 & 0.5 & 1.0 & 0.5 & 45 & 0.2 & 1.278982 & 0.170260 \\
10.0 & 10.0 & 0.71 & 0.22 & 2.0 & 0.5 & 1.0 & 0.5 & 30 & 0.3 & 1.322450 & 0.207208 \\
\hline
\end{tabular}

Table 4. Numerical values of Nusselt number

\begin{tabular}{|c|c|c|c|}
\hline$P_{r}$ & $R$ & $t$ & $N_{u}$ \\
\hline 0.71 & 1.0 & 0.2 & 0.147746 \\
7.00 & 1.0 & 0.2 & 0.445450 \\
0.71 & 2.0 & 0.2 & 0.120620 \\
0.71 & 1.0 & 0.3 & 0.088648 \\
\hline
\end{tabular}

Table 5. Numerical values of Sherwood number

\begin{tabular}{|c|c|c|c|}
\hline$S_{c}$ & $\gamma$ & $t$ & $S_{h}$ \\
\hline 0.22 & 0.5 & 0.2 & 0.262394 \\
0.62 & 0.5 & 0.2 & 0.433564 \\
0.22 & 1.0 & 0.2 & 0.290562 \\
0.22 & 0.5 & 0.3 & 0.229272 \\
\hline
\end{tabular}

\section{Conclusions}

In this paper, the unsteady MHD mixed convective radiating and chemically reacting fluid flow past an impulsively started oscillating vertical plate with Hall current is provided. The finite element method has been applied to solve the dimensionless governing equations of the flow. It has been found that thermal and mass buoyancy force, Hall parameter, radiation parameter and time tends to accelerate both $u$ and $w$ whereas an increase in Prandtl number, Schmidt number, and chemical reaction rate tends to decelerate both $u$ and $w$. Increase in the magnetic parameter depreciate $u$ and reverse trend is noticed on $w$. These parameters have similar effect on both primary and secondary skin frictions. Phase angle causes to decrease both $u$ and $w$, and opposite effect is noticed on the primary and secondary skin frictions. The fluid temperature enhanced with increment in radiation parameter and time whereas reverse trend is noticed when Prandtl number is increased and opposite effect is noticed on the Nusselt number. The fluid concentration decline with increment in Schmidt number and chemical reaction rate whereas opposite trend is observed with progression of time and opposite effect is noticed on the Sherwood number.

\section{Nomenclature}

$u^{\prime} \quad$ Velocity of the fluid in $x^{\prime}-$ direction

$w^{\prime} \quad$ Velocity of the fluid in $z^{\prime}-$ direction

$g \quad$ Acceleration due to gravity

$\beta \quad$ Volumetric coefficient of thermal expansion

$\beta^{*} \quad$ Volumetric coefficient of concentration expansion

$C^{\prime} \quad$ Species concentration

$C_{\infty}^{\prime} \quad$ Concentration in the fluid away from the plate 


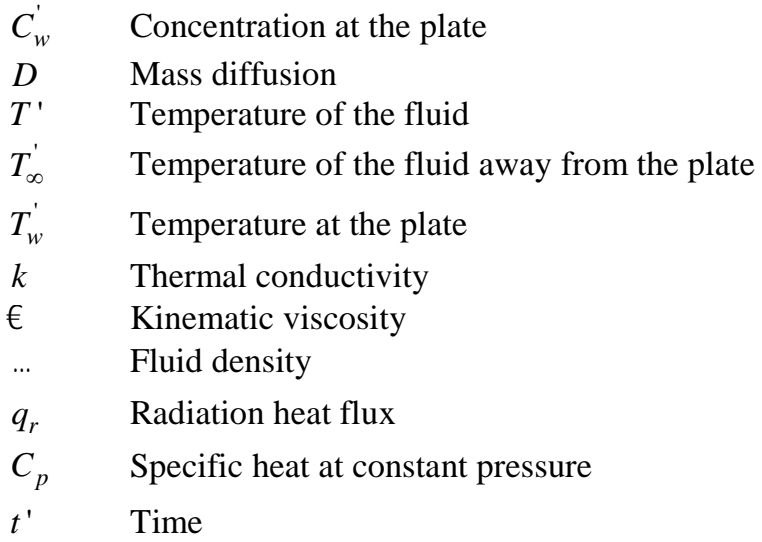

\section{Acknowledgement}

The author's are very grateful to the editor and reviewers for the constructive comments that led to the fruitful improvement in the manuscript.

\section{References}

Bathe, K. J. 1996. Finite element procedures, Prentice-Hall, New Jersey.

Cowling, T. C. 1957. Magnetohydrodynamics, Wiley Inter Science, New York.

El-Fayez, F. M. N. 2012. Effects of chemical reaction on the unsteady free convection flow past an infinite vertical permeable moving plate with variable temperature, Journal of Surface Engineered Materials and Advanced Technology, Vol.2, pp.100 109.

Jayakar, R., Kumar, B. and Makinde, O. D. 2018. Thermo-diffusion effects on MHD chemically reacting fluid flow past an inclined porous plate in a slip flow regime, Defect and Diffusion forum, Vol.387, pp. 587 - 599.

Muthucumaraswamy R. and Janakiraman, B. 2006. MHD and radiation effects on moving isothermal vertical plate with variable mass diffusion, Theoretical and Applied Mechanics, Vol.33, No.1, pp.17 - 29.

Muthucumaraswamy, R. and Meenakshisundaram, S. 2006. Theoretical study of chemical reaction effects on vertical oscillating plate with variable temperature, Theoretical and Applied Mechanics, Vol.33, No.3, pp.245 - 257.

Muthucumaraswamy, R. and Janakiraman, B. 2008. Mass transfer effects on isothermal vertical oscillating plate in the presence of chemical reaction, International Journal of Applied Mathematics and Mechanics, Vol.4, No.1, pp. 66 - 74.

Mahapatra, N., Dash, G. C., Panda, S. and Acharya, M. 2010. Effects of chemical reaction on free convection flow through a porous medium bounded by a vertical surface, Journal of Engineering Physics and Thermophysics, Vol.83, No.1, pp.130 - 140.

Magyari, E. and Pantokratoras, A. 2011. Note on the effect of thermal radiation in the linearized Rosseland approximation on the heat transfer characteristics of various boundary layer flows, International Communications in Heat and Mass Transfer, Vol.38, pp. 554-556

Mebine. P. and Adigio E. M. 2011. Effects of thermal radiation on transient MHD free convection flow over a vertical surface embedded in a porous medium with periodic boundary conditions, Mathematica Aeterna - International Journal for Pure and Applied Mathematics, Vol.I, pp.245 - 261.

Makinde, O. D. 2012. Chemically reacting hydro-magnetic unsteady flow of radiating fluid past a vertical plate with constant heat flux, Zeitschrift f'ur Naturforschung, Vol.67a, pp.239 - 247.

Maguna, A. N. and Mutua, N. M. 2013. Hall current effects on free convection flow and mass transfer past a semi infinite vertical flat plate, International Journal of Mathematics and Statistics Studies, Vol. 1, No.4, pp. 1 - 22.

Muhammad, A. and Makinde, O. D. 2017. Thermo-dynamic analysis of unsteady MHD mixed convection with slip and thermal radiation over a permeable surface, Defect and Diffusion Forum, Vol.374, pp. 29 - 46.

Nandkeolyar, R., Seth, G. S., Makinde, O. D., Sibanda, P and Ansari, M. S. 2013. Unsteady hydro-magnetic natural convection flow of a dusty fluid past an impulsively moving vertical plate with ramped temperature in the presence of thermal radiation, ASME, Journal of Applied Mechanics, Vol.80, 061003, pp.1 - 90.

Prabhakar Reddy, B. 2018. Hall effect on MHD transient flow past an impulsively started infinite horizontal porous plate in a rotating system, International Journal of Applied Mathematics and Mechanics, Vol. 23, No.2, pp.471 - 483.

Reddy, J. N. 1985. An introduction to the finite element method, McGraw-Hill, New York.

Rajesh, V. and Varma, S. V. K. 2010. Radiation effects on MHD flow through a porous medium with variable temperature and mass diffusion, International Journal of Applied Mathematics and Mechanics, Vol.6, No.1, pp.39 - 57.

Rajesh, V. and Varma, S. V. K. 2010. Chemical reaction effects on free convection flow past an exponentially accelerated vertical plate, Annals-Journal of Engineering, VIII, Fascicule 1, pp. 181 - 188. 
Rajput, U. S. and Kanaujia, N. 2016. MHD flow past a vertical plate with variable temperature and mass diffusion in the presence of Hall current, International Journal of Applied Sciences and Engineering, Vol.14, No.2, pp. 115 - 123.

Rajput, U. S. and Kumar, G. 2017. Radiation effect on MHD flow past an inclined plate with variable temperature and mass diffusion, International Journal of Applied Sciences and Engineering, Vol.14, No.3, pp. 171 - 183.

Sharma, B. K., Jha, A. K and Chaudhary, R. C. 2007. Hall effect on MHD mixed convection flow of a viscous incompressible fluid past a vertical porous plate immersed in a porous medium with heat source/sink, Romanian Journal of Physics, Vol.52, No.5-7,pp. $487-503$.

Sehkar, D. V. and Reddy, G. V. 2012. Effects of chemical reaction on MHD free convective oscillatory flow past a porous plate with viscous dissipation and heat sink, Advances in Applied Science Research, Vol.3, No.5, pp.3206 - 3215.

Thamizhsudar, M., Pandurangan, J. and Muthucumaraswamy, R. 2015. Hall effects and rotation effects on MHD flow past an exponentially accelerated vertical plate with combined heat and mass transfer effects, International Journal of Applied Mechanics and Engineering, Vol.20, No. 3, pp. 605 - 616.

Zienkiewiez, O. C. 1971. The Finite Element Method in Engineering Sciences - $2^{\text {nd }}$ Edition., McGraw-Hill, New York.

\section{Biographical notes}

P.M. Matao is currently working as assistant Lecturer in the Department of Mathematics and Statistics, CNMS, The University of Dodoma, P.O Box No: 338 Dodoma, Tanzania. He is pursuing $\mathrm{PhD}$ studies in the area of computational fluid dynamics. He has published two research papers and presented two papers in International conferences.

Dr. B. Prabhakar Reddy currently working as a senior lecturer in the Department of Mathematics and Statistics, CNMS, The University of Dodoma, P.O Box No: 338, Dodoma, Tanzania. He received his PhD in Mathematics from Osmania University, Hyderabad, Telangana, India. He has more than 20 years of teaching experience for both UG and PG level courses and around 10 years of research studies. His current area of research includes fluid Dynamics, MHD, Heat and mass transfer flow problems by FDM and FEM. He has published more than 40 research papers in National/International journals of repute.

Dr. Jefta M. Sunzu currently working as a Head Department of Mathematics and Statistics, CNMS, The University of Dodoma, P.O Box No: 338, Dodoma, Tanzania. He received his PhD in Mathematics from KwaZulu Natal University, Soth Africa, in the year 2014. He has more than 10 years of teaching experience for both UG and PG courses and around 8 years of research studies. His current area of research includes Austro physics, Financial Mathematics and fluid Dynamics. He has published several research papers in National/International journals of repute.

O. D. Makinde is currently a Professor of Computational and Applied Mathematics at the Faculty of Military Science, Stellenbosch University, South Africa. He is a visiting Professor to several other Universities. He is a Fellow of African Academy of Sciences, Fellow of International Academy of Physical Sciences and Fellow of Papua New Guinea Mathematical Society. He won several prestigious academic research awards and honours including: African Union Kwame Nkrumah Continental Scientific Award; South African TW Kambule Senior Researcher Award, Stellenbosch University Chancellor Award and Nigerian National Honour Award - Member of Order of the Federal Republic (MFR). He is a reviewer, editor and editorial board member of several international journals in the frame of Engineering Science, Applied Mathematics and Computations. His main research interests are: fluid mechanics, nanofluid dynamics, heat and mass transfer, hydrodynamic stability, mathematical modelling, computational mathematics, biomathematics and applications.

Received September 2019

Accepted December 2019

Final acceptance in revised form December2019 